\title{
Granulomatosis with polyangiitis presenting with pachymeningitis and persistent headache
}

\author{
Maria Sofia Cotelli, ${ }^{1}$ Mirko Scarsi, ${ }^{2}$ Marta Bianchi, ${ }^{1}$ Valeria Bertasi, ${ }^{1}$ Patrizia Civelli, ${ }^{1}$ Filippo Manelli, ${ }^{3}$ \\ Marinella Turla ${ }^{1}$ \\ ${ }^{1}$ Neurology Unit; ${ }^{2}$ Internal Medicine Unit; and ${ }^{3}$ Emergency Unit, ASST Valcamonica Esine, Brescia, Italy
}

\begin{abstract}
Granulomatosis with polyangiitis (GPA), formerly known as Wegener's granulomatosis, is a systemic inflammatory disease, primarily involving the upper and lower respiratory tract and kidneys. It is a relatively uncommon condition, characterized by necrotizing granulomatous vasculitis of small- and medium-sized vessels and the presence of anti-neutrophil cytoplasmic antibodies in the serum as defined in the Chapel Hill Consensus Conference 2012. GPA presents a wide spectrum of manifestations and remains one of the most challenging diagnostic dilemmas in clinical medicine. From common respiratory and neurological symptoms to infrequent cardiac complications, this fatal systemic illness is difficult to distinguish from infectious etiologies, and it is often mistaken for an isolated complaint.

We report a case of granulomatosis with polyangiitis in a patient with persistent frontal headache, for a very long time considered as migraine versus tension-type headache.
\end{abstract}

\section{Case Report}

We report the case of a 61-year-old who man was hospitalized due to the presence of persistent fever and left orbital swelling from about one week. He also complained of left orbital headache, which described as pressing and tightening in quality, of mild to moderate intensity, daily persistent from about one year. He had undergone several neurological evaluations by different Colleagues, who suggested treatment with prophylactic molecules such as amitriptyline, topiramate, propranolol, flunarizine without any benefits.

Correspondence: Maria Sofia Cotelli, Neurology Unit, ASST Valcamonica Esine, Brescia, Italy.

E-mail: cotellim@gmail.com

Key words: Granulomatosis; headache; vasculitis; pachymeningitis.

Contributions: all authors contributed equally to this work.

Conflict of interests: all authors declare no conflict of interests.

Received for publication: 22 June 2019.

Revision received: 17 October 2019.

Accepted for publication: 23 October 2019.

This work is licensed under a Creative Commons Attribution NonCommercial 4.0 License (CC BY-NC 4.0).

${ }^{\circ}$ Copyright: the Author(s), 2020

Licensee PAGEPress, Italy

Italian Journal of Medicine 2020; 14:27-30

doi:10.4081/itjm.2020.1193
He used to take non-steroidal anti-inflammatory drugs only when pain was unbearable.

He had performed axial brain tomography and paranasal sinuses attesting possible sinusopathy of the maxillary sinuses and diffuse subdural nodulations, for which he had been treated with unspecified antibiotic therapy and, for about 14 days, with steroid with extreme benefit on pain (oral methylprednisolone 25 $\mathrm{mg}$ /day). However, symptoms promptly relapsed after steroid withdrawal. His pathological history was positive for bronchial asthma since 2015, and previous diagnosis of essential thrombocythemia with JAK-2 (V617) mutation started with Budd-Chiari syndrome in 2011, for which a jugular portosystemic shunt had been introduced and anticoagulant therapy with warfarin had been started. A brain computer tomography performed in 2015 showed left frontal chronic subdural hematoma (which had disappeared after one month).

\section{Results}

Neurological examination during hospitalization (with special attention to cranial nerves or meningeal signs) remained normal. However, he persisted in complaining of persistent headache (visual analogue scale of about $8 / 10$ ) with nausea and photophobia, and evidence of left eyelid swelling and red eye. Blood exams showed high white blood cells count $\left(15.840 \times 10^{9} / \mathrm{L}\right)$, elevate values of reactive $\mathrm{C}$ protein $(82.8 \mathrm{~g} / \mathrm{L})$, alkaline phosphatase $(168 \mathrm{UI} / \mathrm{L}$ normal value $40-150$ ), alanine amino transferase ( $89 \mathrm{UI} / \mathrm{L}$ normal value $<55)$, gamma-glutamyl transferase (196 $\mathrm{UI} / \mathrm{L}$ normal value $<55$ ), while anti neutrophil cyto- 
plasmic antibodies resulted +/-. The results for antinuclear antibodies, human immunodeficiency virus, hepatitis B surface antigen immunological panel were all negative.

He underwent brain magnetic resonance (liver acquisition with volume acquisition sequences) attesting signal alteration of the paranasal walls which resulted thickened with involvement of the orbital walls, infiltration along pterygopalatine fossa, cavernous sinuses, Meckel ganglion, maxillary branch of trigeminal nerve bilaterally and left maxillary branch and of dural, tentorial, retroclival, orbital, frontal regions (Figures 1 and 2). Neuroradiological pattern was suggestive of granulomatous pachymeningitis. He underwent nasal biopsy attesting to acute erosive mucositis with evident hypereosinophilia and granulomas, chest tomography (not specific signs attributable to granulomatosis), echocardiogram attesting to hypertrophic non-obstructive cardiomyopathy with pericardial effusion.

High-dose prednisone (prednisone $62.6 \mathrm{mg} /$ day) was started. Due to the evidence of the nasal swab for Staphylococcus aureus and Pseudomonas aeruginosa, trimethropim-sulfamethoxazole was initiated and antipneumococcal vaccination with a 23-polyvalent vaccine was promptly administered. On the basis of the European League Against Rheumatism (EULAR) guidelines, the rheumatology colleagues carried out the single administration of cyclophosphamide 15 $\mathrm{mg} / \mathrm{kg}=900 \mathrm{mg}$ after pre-and post-premedication with sodium mercaptoethanesulfonate and ondansetron. The patient had no complications and the headache disappeared while the orbital swelling resulted visibly reduced. He was discharged with a diagnosis of Wegener's granulomatosis, with steroid therapy admin- istered as a diagnosis and co-trimoxazole $1000 \mathrm{mg}$ every 2 days.

\section{Discussion}

Granulomatosis with polyangiitis is a rare form of necrotizing granulomatous vasculitis, typically involving the nasal cavity, lung, and kidney as a triad, although it can affect any organ systems. ${ }^{1}$ More than $90 \%$ of patients initially present with upper or lower airway symptoms or both. ${ }^{2}$ The overall annual incidence of GPA is approximately $2.4-11.3$ cases per one million with no significant gender predilection. ${ }^{3}$ The age of symptom onset has a wide distribution, with peak incidence between the ages of 41 and 68 years, and a very rare occurrence in childhood and young adults. ${ }^{3}$ There is a significantly higher prevalence of GPA amongst Caucasians, especially those from Northern Europe, compared to Asian and African populations. ${ }^{3}$ These differences may be attributed to differences in geographical, environmental, and genetic factors. ${ }^{3}$

GPA's complex multifactorial etiology is indicated to result from immunologic and environmental contributions in genetically predisposed individuals. Genetic predisposition amongst GPA patients is heterogeneous and may result in variations in human leukocyte antigen-mediated antigen presentation, a defective immune regulation, and an abnormal structure and function of the target antigen. ${ }^{4}$

Neurological involvement during the course of the disease is usually in the form of mononeuritis multiplex and cranial neuropathy. Headache, cerebritis, seizures, stroke, meningitis, diabetes insipidus, hydro-

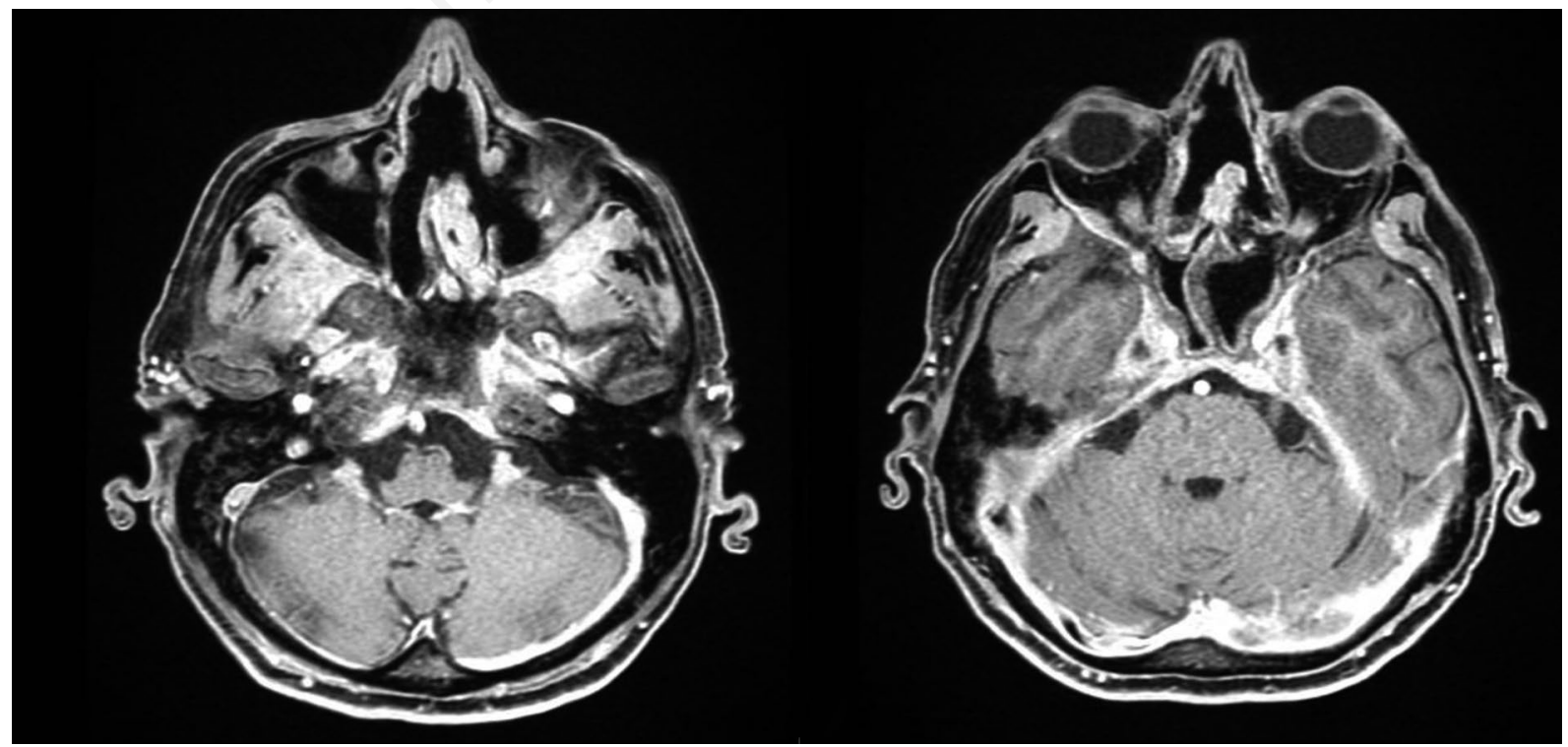

Figure 1. Axial liver acquisition with volume acquisition sequence. 
cephalus, external ophthalmoplegia, myelopathy, and myopathy are other manifestations which are seen less frequently. ${ }^{5}$ Central nervous system (CNS) involvement usually occurs during the later stages of the disease, and dural enhancement has been recognized as one of the well-known findings on magnetic resonance images. ${ }^{6}$ The involvement of the CNS has been reported in 8 to $54 \%$ of patients with Wegener's granulomatosis (WG), ${ }^{7}$ and the modes of presentation include contiguous granulomatous invasion from the nasal cavity, cerebral vasculitis, ${ }^{5}$ and peripheral neuropathy. ${ }^{8}$ Nonetheless, involvement of the CNS at the time of the patient initial presentation is considered rare (1-2\%). ${ }^{9}$ Three mechanisms are involved in the pathogenesis of the different CNS manifestations seen in WG: i) vasculitis of the nervous system; ii) contiguous invasion of granuloma from extracranial sites; iii) and, remote intracranial granuloma. ${ }^{5}$

Patients with clinically active granulomatosis with polyangiitis may present with constitutional symptoms of disease such as general malaise, myalgia, arthralgia, anorexia, weight loss and pyrexia. ${ }^{10}$

Other symptoms can be cutaneous (such as leukocytoclastic vasculitis, digital infarcts, purpura, cutaneous ulcers and gangrene) mucocutaneous (which include oral ulcers, oral granulomatous lesions, episcleritis, scleritis, conjunctivitis, keratitis, uveitis, retinal vasculitis, retinal arterial or venous thrombosis, retinal exudates, retinal hemorrhages, blurred vision, blindness, proptosis and orbital granulomatous masses), ear, nose and upper airway, which are common amongst GPA patients (such as sensorineural hearing loss and conductive hearing persistent, recurrent nasal discharges, blood-stained nasal discharge, epistaxis, nasal crusting, nasal ulceration, nasal bridge collapse, nasal granulomatous lesions, parasinus and

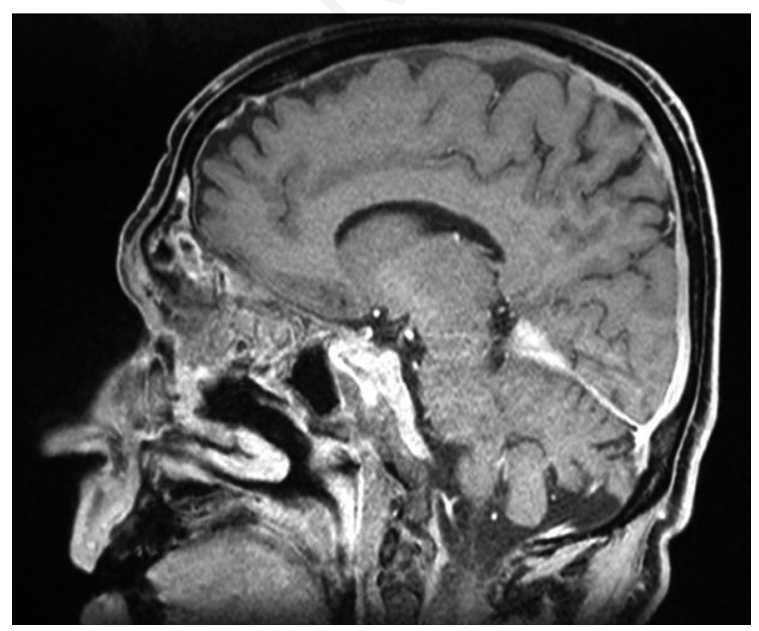

Figure 2. Sagittal liver acquisition with volume acquisition sequence. sinus inflammation),${ }^{10}$ lower respiratory tract manifestations (cough, breathlessness, stridor, wheeze, small airway obstruction, pulmonary nodules, cavitating lung lesions, pleuritis, pleural effusions, pulmonary infiltrates, pulmonary hemorrhage, due to alveolar capillaritis and respiratory failure), cardiovascular (small vessel vasculitis, occlusive vascular disease, pericarditis, pericardial effusions, cardiomyopathy, valvular heart disease, ischemic heart disease and heart failure), gastrointestinal, renal (pauci-immune crescentic necrotizing glomerulonephritis). ${ }^{11}$

CNS and peripheral nervous system GPA, has a variety of clinical presentations which include headache, meningitis, seizures, cerebrovascular accidents, spinal cord lesions, cranial nerve palsies, sensory or motor peripheral neuropathy, mononeuritis multiplex; sensorineural hearing loss and cerebral mass lesions. ${ }^{11}$

GPA conventional treatment relies mainly on agents that decrease inflammation: corticosteroids and immunosuppressant adjunction for severe manifestations. New therapeutic approaches are needed for refractory disease, relapses and issues associated with corticosteroid dependence, especially for asthma manifestations. ${ }^{12}$ Drugs under evaluation mostly target eosinophils and B cells. Results of low-evidencebased trials suggested possible efficacies of biologicals: B-cell-blocking rituximab and anti-immunoglobulin E omalizumab. ${ }^{12}$ Recently, the first large-scale randomized controlled trial on eosinophilic granulomatosis with polyangiitis proved the efficacy of anti-interleukin-5 mepolizumab. ${ }^{12}$

\section{Conclusions}

Our patient presents a medical history positive for essential thrombocythemia JAK2 with Budd Chiari syndrome (generally not associated with pachymeningitis). He had received diagnoses of migraine without aura, tension-type headache and chronic daily headache for about one year and had been treated only with prophylactic treatments for headache without any benefits. We think that it would have been better not to wait to perform neuroimaging exams, considering his previous medical history, the relatively recent appearance of headache, and the prompt response only to steroid with complete failure of all prophylaxis attempts. We did not perform lumbar puncture due to anticoagulation therapy that the patient was taking and decided to start immediately with immunosuppressants after discussing magnetic resonance imaging with neuroradiologists (who considered it compatible with WG).

In conclusion, when evaluating patients with atypical headache responsive only to steroid therapy, it should be useful to consider autoimmune diseases as a possible differential diagnosis with chronic daily 
headache, especially in the presence of atypical response to classic drugs.

\section{References}

1. Leavitt RY, Fauci AS, Bloch DA, et al. The American College of Rheumatology 1990 criteria for the classification of Wegener's granulomatosis. Arthritis Rheum 1990;33:1101-7.

2. Hoffman GS, Kerr GS, Leavitt RY, et al. Wegener granulomatosis: an analysis of 158 patients. Ann Intern Med 1992;116:488-98.

3. Grygiel-Górniak B, Limphaibool N, Perkowska B, et al. Clinical manifestations of granulomatosis with polyangiitis: key considerations and major features. M Postgrad Med 2018;130:581-96.

4. Cartin-Ceba R, Peikert T, Specks U. Pathogenesis of ANCA-associated vasculitis. Rheum Dis Clin North Am 2010;36:463-77.

5. Jinnah HA, Dixon A, Brat DJ, Hellmann DB. Chronic meningitis with cranial neuropathies in Wegener's gran- ulomatosis. Case report and review of the literature. Arthritis Rheum 1997;40:573-7.

6. Drachman DA. Neurological complications of Wegener's granulomatosis. Arch Neurol 1963;8:145-55.

7. Fauci AS, Haynes BF, Katz P, et al. Wegener's granulomatosis: prospective clinical and therapeutic experience with 85 patients for 21 years. Ann Intern Med 1983; 98:76-85.

8. Hoffman GS, Kerr GS, Leavitt RY, et al. Wegener granulomatosis: an analysis of 158 patients. Ann Intern Med 1992;116:488-98.

9. Gupta V, Sharma AK, Sureka RK, et al. Chronic meningitis with multiple cranial neuropathies: A rare initial presentation of Wegener's granulomatosis. Ann Indian Acad Neurol 2013;16:411-3.

10. Lutalo PM, D'Cruz DP. Diagnosis and classification of granulomatosis with polyangiitis (aka Wegener's granulomatosis). J Autoimmun 2014;48-49:94-8.

11. Seo P, Stone JH. The antineutrophil cytoplasmic antibody-associated vasculitides. Am J Med 2004;117:39e50.

12. Raffray L, Guillevin L Treatment of eosinophilic granulomatosis with polyangiitis: a review. Drugs 2018;78: 809-21. 